\title{
Mesoscopic Kinetic Basis of Macroscopic Chemical Thermodynamics: A Mathematical Theory
}

\author{
Hao $\mathrm{Ge}^{1 *}$ and Hong Qian ${ }^{2 \dagger}$ \\ ${ }^{1}$ Beijing International Center for Mathematical Research (BICMR) \\ Biodynamic Optical Imaging Center (BIOPIC) \\ Peking University, Beijing 100871, P.R.C. \\ ${ }^{2}$ Department of Applied Mathematics, University of Washington \\ Seattle, WA 98195-3925, U.S.A
}

January 14, 2016

\begin{abstract}
From a mathematical model that describes a complex chemical kinetic system of $N$ species and $M$ elementrary reactions in a rapidly stirred vessel of size $V$ as a Markov process, we show that a macroscopic chemical thermodynamics emerges as $V \rightarrow \infty$. The theory is applicable to linear and nonlinear reactions, closed systems reaching chemical equilibrium, or open, driven systems approaching to nonequilibrium steady states. A generalized mesoscopic free energy gives rise to a macroscopic chemical energy function $\varphi^{s s}(\mathbf{x})$ where $\mathbf{x}=\left(x_{1}, \cdots, x_{N}\right)$ are the concentrations of the $N$ chemical species. The macroscopic chemical dynamics $\mathbf{x}(t)$ satisfies two emergent laws: (1) $(\mathrm{d} / \mathrm{d} t) \varphi^{s s}[\mathbf{x}(t)] \leq 0$; and (2) $(\mathrm{d} / \mathrm{d} t) \varphi^{s s}[\mathbf{x}(t)]=$ $\operatorname{cmf}(\mathbf{x})-\sigma(\mathbf{x})$ where entropy production rate $\sigma \geq 0$ represents the sink for the chemical energy, and chemical motive force $\mathrm{cmf} \geq 0$ is non-zero if the system is driven under a sustained nonequilibrium chemostat. For systems with detailed balance $\mathrm{cmf}=0$; and if one assumes the law of mass action, $\varphi^{s s}(\mathbf{x})$ is precisely the Gibbs' function $\sum_{i=1}^{N} x_{i}\left[\mu_{i}^{o}+\ln x_{i}\right]$
\end{abstract}

*haoge@pku.edu.cn

†hqian@u.washington.edu 
for ideal solutions. For a class of kinetic systems called complex balanced, which include many nonlinear systems as well as many simple open, driven chemical systems, the $\varphi^{s s}(\mathbf{x})$, with global minimum at $\mathbf{x}^{*}$, has the generic form $\sum_{i=1}^{N} x_{i}\left[\ln \left(x_{i} / x_{i}^{*}\right)-x_{i}+x_{i}^{*}\right]$, which has been known in chemical kinetic literature. Macroscopic emergent "laws" are independent of the details of the underlying kinetics. This theory provides a concrete example from chemistry showing how a dynamic macroscopic law can emerge from the kinetics at a level below.

Ever since the work of J. W. Gibbs and the influential treatise of Lewis and Randall [1], chemical thermodynamics has been one of the most important theoretical cornerstones of chemical science. While temperature is one of the key concepts in the theory, its origin resides in the mechanical movement of atoms and molecules, as already clearly articulated by L. Boltzmann in his mechanical theory of heat [2]. The notion of chemical potential, however, can not be understood from Newtonian mechanics. This is best illustrated through its definition in Gibbs' equation:

$$
\mathrm{d} U=T \mathrm{~d} S-p \mathrm{~d} V+\sum_{i=1}^{n} \mathrm{~d} \mu_{i} N_{i}
$$

in which

$$
T=\left(\frac{\partial U}{\partial S}\right)_{V,\left\{N_{i}\right\}}, p=-\left(\frac{\partial U}{\partial V}\right)_{S,\left\{N_{i}\right\}}, \mu_{i}=\left(\frac{\partial U}{\partial N_{i}}\right)_{S, V,\left\{N_{j, j \neq i}\right\}}
$$

In graduate texts on Newtonian mechanics, there is a demonstration of that [3] $\partial U / \partial S$ is the mean kinetic energy if one identifies the $S \equiv k_{B} \ln \Omega$ as the phase volume of a Hamiltonian dynamics; and $-\partial U / \partial V$ as the momentum transfer on the wall of a box that contains gas particles. While these demonstrations are not general, they have provided definitive mechanical interpretations of the two emergent thermodynamic quantities. On the contrary, there is no mechanical interpretation for the $\partial U / \partial N$ in (2). It is widely felt that a "mechanistic" interpretation of the chemical potential $\mu_{i}$ has to be a probabilistic one. We hasten to mention that a living organism is sustained as a nonequilibrium system neither by a difference in $T$ nor $p$, rather it is a phenomenon driven by $\Delta \mu$. Therefore, a fundamental understanding of the irreversibility of life requires a deeper understanding of $\mu$, which is still lacking a rigorous sub-macroscopic foundation.

The description of the mathematical model. We report in this note a recently discovered mathematical result that provides the chemical potential $\mu$, as a emergent macroscopic quantity, an origin in a mesoscopic description of chemical kinetics. The mesoscopic description of a chemical kinetics is based on a system of elementary chemical reactions with arbitrary complexity; reactions occur one 
at a time in a stochastic fashion, as now clearly demonstrated in single-molecule studies [4]. The general setting has $N$ chemical species and $M$ reactions in a fixed volume of $V$ [5]:

$$
\nu_{\ell 1}^{+} X_{1}+\nu_{\ell 2}^{+} X_{2}+\cdots \nu_{\ell N}^{+} X_{N} \underset{k_{-\ell}}{\stackrel{k_{+\ell}}{\rightleftharpoons}} \nu_{\ell 1}^{-} X_{1}+\nu_{\ell 2}^{-} X_{2}+\cdots \nu_{\ell N}^{-} X_{N},
$$

in which $1 \leq \ell \leq M . \nu_{i j}=\left(\nu_{i j}^{-}-\nu_{i j}^{+}\right)$are the stoichiometric coefficients relating species to reactions. In a reaction vessel with rapidly stirred chemical solutions, the numbers of species $i$ at time $t$ is denoted by $n_{i}(t)$. Our theory assumes that

(i) Each reaction is microscopically reversible, with forward rate $r_{+\ell}(\mathbf{n}(t))$ and backward rate $r_{-\ell}(\mathbf{n}(t))$ where $\mathbf{n}=\left(n_{1}, \cdots, n_{N}\right)$ denoting the copy numbers of all the species. Both $r_{ \pm \ell}$ are mathematically non-negative; but their dependences on $\mathbf{n}$ are essentailly arbitrary (with some minor mathematical assumptions).

(ii) As an elementrary reaction, each occurs as a Markov process with exponential waiting time following the distribution $r(\mathbf{n}) e^{-r(\mathbf{n}) t}$.

(iii) The mesoscopic rate $r_{+\ell}(\mathbf{n})$ is the number of occurrences of the $\ell^{\text {th }}$ forward reaction per unit time in the volume $V$. Therefore, for a macroscopic system with extremely large $\mathbf{n}$ and $V:\left(x_{1}, \cdots, x_{N}\right) \equiv \mathbf{x}=\mathbf{n} / V$ are the concentrations, and $R_{ \pm \ell}(\mathbf{x})=r_{ \pm \ell}(V \mathbf{x}) / V$ are the concentration-based rates of the reactions.

(iv) The chemical reaction system can be either closed or open. A closed system has no exchange of matter with its surrounding; an open system can exchange various chemical species with its surrounding, which are kept at constant concentrations. If all the "externally buffered" species are themselves at a chemical equilibrium, the situation is like a dialysis system, which ultimately reach a chemical equilibrium both within and with its surrounding. If, however, there are at least two species that are sustained at a nonequilibrium condition, then the system eventually settles in a nonequilibrium state with stationary concentration fluctuations. This last scenario is the biochemical kinetic setup for modeling a living cells under a continuously chemostat.

With these rather general assumptions, T. G. Kurtz has shown in 1972 [6] that in the limit of $V \rightarrow \infty$, the mesoscopic stochastic description of the system of chemical reactions becomes the following set of rate equations for the macroscopic kinetics:

$$
\frac{\mathrm{d} x_{i}(t)}{\mathrm{d} t}=\sum_{\ell=1}^{M} \nu_{\ell i}\left(R_{+\ell}(\mathbf{x})-R_{-\ell}(\mathbf{x})\right),
$$

$1 \leq i \leq N$. Kurtz's theorem paves the way for a unified mathematical theory of chemical kinetics in a rapidly stirred vessel of both small and large size.

Based on such Markov processes, a mesoscopic statistical (or stochastic) thermodynamic theory has been developed in recent years in the field of nonequi- 
librium statistical physics. The most celebrated results from this theory is the Jarzynski-Crooks equality [7, 8]. The mesoscopic theory also, for the first time, demonstrated a free energy blanace equation: One can introduce a generalized, nonequilibrium free energy $F^{(m e s o)}$ for any chemical reaction systems at the mesoscopic level. Then it can be mathematically shown that this $F^{(m e s o)}$ satisfied an instantaneous balance relation [9, 10, 11]:

$$
\frac{\mathrm{d} F^{(m e s o)}}{\mathrm{d} t}=E_{i n}-e_{p}
$$

in which both $E_{i n}$ and $e_{p}$ are non-negative, and $d F^{(m e s o)} / d t$ is never positive. When applied to the chemomechanics of a single ATPase motor protein, the $E_{\text {in }}$ is the amount of chemical energy input per unit time, e.g., ATP hydrolysis, and $e_{p}$ is the minimal amount of heat dissipation [12]. If the motor is actually performing mechanical work against an external elastic force $f^{e x t}$ and moving with velocity $v$, then both $E_{i n}$ and $e_{p}$ contains the mechanical power $f^{(e x t)} v$.

For a closed chemical reaction system, or an open system in contact with a single external chemical potential $\mu^{e x t}$, it can be shown that $E_{i n}=0$. In this case, the $F^{(m e s o)}$ is indeed the free energy of the molecular system [13, 14]. Actually, with the help of detailed balance, the stochastic Markov theory and Gibbs' canonical and grand canonical emsemble theories are proven equivalent.

The kinetic theory of macroscopic chemical (free) energy. We summarize the new mathematical results [15, 16]. So far, the equation is only establised in the mesoscopic setting. So all three non-negative quantities, entropy production $e_{p}$, energy input $E_{i n}$, and free energy dissipation $-d F^{(m e s o)} / d t$ are functions of the volume parameter $V$. Furthermore, they are functions of the probability distribution of the number of each species at time $t, p_{V}(\mathbf{n}, t)$, which itself is also a function of $V$.

Now if we perform the limit of $V \rightarrow \infty$, Kurtz's theorem tells us that $\mathbf{x}(t)=$ $\mathbf{n}(t) / V$ is the solution to the nonlinear rate equation (4). Furthermore, the probability theory also suggests that for a wide class of models, $p_{V}(V \mathbf{x}, t)$ can be written as $\exp (-V \varphi(\mathbf{x}, t))$, and in the stationary state a $\varphi^{s s}(\mathbf{x})$ emerges, which satisfies the following equation, derived independently by Kurtz in 1978 [17] and G. Hu in 1986 [18]

$$
\sum_{\ell=1}^{M} R_{+\ell}(\mathbf{x})\left[1-e^{\boldsymbol{\nu}_{\ell} \cdot \nabla_{\mathbf{x}} \varphi^{s s}(\mathbf{x})}\right]+R_{-\ell}(\mathbf{x})\left[1-e^{-\boldsymbol{\nu}_{\ell} \cdot \nabla_{\mathbf{x}} \varphi^{s s}(\mathbf{x})}\right]=0
$$

where $\boldsymbol{\nu}=\left(\nu_{1}, \cdots, \nu_{M}\right)$. We have shown that in the "macroscopic limit", as intensive quantities

$$
V^{-1} F^{(m e s o)} \rightarrow \varphi^{s s}(\mathbf{x}),
$$




$$
\begin{gathered}
\frac{\mathrm{d} \varphi^{s s}(\mathbf{x})}{\mathrm{d} t}=\operatorname{cmf}(\mathbf{x})-\sigma(\mathbf{x}) \\
V^{-1} e_{p} \rightarrow \sigma(\mathbf{x})=\sum_{\ell=1}^{M}\left(R_{+\ell}(\mathbf{x})-R_{-\ell}(\mathbf{x})\right) \ln \left(\frac{R_{+\ell}(\mathbf{x})}{R_{-\ell}(\mathbf{x})}\right) \\
V^{-1} E_{i n} \rightarrow \operatorname{cmf}(\mathbf{x})=\sum_{\ell=1}^{M}\left(R_{+\ell}(\mathbf{x})-R_{-\ell}(\mathbf{x})\right) \ln \left(\frac{R_{+\ell}(\mathbf{x})}{R_{-\ell}(\mathbf{x})} e^{\nu \cdot \nabla_{\mathbf{x}} \varphi^{s s}(\mathbf{x})}\right)
\end{gathered}
$$

A macroscopic chemical energy function $\varphi^{s s}(\mathbf{x})$ emerges. For the stationary state with large but finite $V$, the probability distribution for concentration fluctuations attains a universal expression

$$
f(\mathbf{x} \mid V)=\frac{\Omega(\mathbf{x}) e^{-V \varphi^{s s}(\mathbf{x})}}{\Xi(V)}, \text { where } \Xi(V)=\int \Omega(\mathbf{x}) e^{-V \varphi^{s s}(\mathbf{x})} \mathrm{d} \mathbf{x},
$$

in which $\Omega(\mathbf{x})$ is analogous to the "degeneracy" in a partition function calculation. As a part of the mathematical theory of large deviations in a probability distribution, Eq. 8 and alike have been considered as the mathematical foundation of equilibrium statistical thermodynamics [19]. Our present work is an application of a dynamic version of such mathematics.

What is the mechanistic force corresponding to the emergent energy function $\varphi^{s s}(\mathbf{x})$ ? More specifically, how does this "force" affect the macroscopic kinetics? First, one needs to consciously recognize the vast separation of time scales in the mesoscopic and macroscopic kinetics. That is why the dynamics of the latter is partially dictated by the stationary behavior of the former, in the form of $\varphi^{s s}(\mathbf{x})$. It is clear that $\varphi^{s s}(\mathbf{x})$ is a consequence of a global, infinitely long time behavior of the mesoscopic system.

Eq. $7 \mathrm{~b}$ is a macroscopic chemical (free) energy balance equation, in which an the emergent chemical motive force (cmf) characterizes the force the environment puts upon the kinetic system, and entropy production rate $\sigma(\mathbf{x})$ characterized the amount of free energy that is dissipated from the system.

Detailed balance and chemical equilibrium. If this global dynamic consequence is in complete consistency with the local kinetic $\ln \left[R_{+\ell}(\mathbf{x}) / R_{-\ell}(\mathbf{x})\right]$, we say the chemical kinetics is in "equilibrium":

$$
\ln \left(\frac{R_{+\ell}(\mathbf{x})}{R_{-\ell}(\mathbf{x})}\right)=\exp \left(-\boldsymbol{\nu}_{\ell} \cdot \nabla_{\mathbf{x}} \varphi_{\mathbf{x}}^{e q}(\mathbf{x})\right), \forall \mathbf{x}
$$

Then $\operatorname{cmf}(\mathbf{x})=0$ for all $\mathbf{x}$, and vice versa. Eq. (9) formalizes the fundamental insights of G. N. Lewis on the importantace of detailed balance in chemical kinetics [20]. In this case there is a chemical equilibrium between the local kinetics and its environment that is created by the other reactions in the same kinetic system. If we 
further assume the Law of Mass Action, then it can be proven mathematically that $\varphi(\mathbf{x})$ is actually the Gibbs function $G(\mathbf{x}) / k_{B} T=\sum_{i=1}^{N} x_{i}\left(\mu_{i}^{o}+k_{B} T \ln x_{i}\right)$, further more Eq. 9 is equivalent to $R_{+\ell}\left(\mathrm{x}^{e q}\right)=R_{-\ell}\left(\mathrm{x}^{e q}\right), \forall \ell$, where $\mathrm{x}^{e q}$ is the unique minimum of $G(\mathbf{x})$. Then Eq. $7 \mathrm{~b}$ becomes $\frac{\mathrm{d} G}{\mathrm{~d} t}=-\sigma(\mathbf{x}) \leq 0$; and $\boldsymbol{\nu}_{\ell} \cdot \nabla_{\mathbf{x}} G(\mathbf{x})=$ $\Delta \mu_{\ell}(\mathbf{x})=k_{B} T \ln \left[R_{-\ell}(\mathbf{x}) / R_{+\ell}(\mathbf{x})\right], \Delta \mu_{\ell}^{o}=\sum_{i=1}^{N} \nu_{\ell i} \mu_{i}^{o}=k_{B} T \ln \left(k_{-\ell} / k_{+\ell}\right)$.

When Eq. 9 is not hold, which is equivalent to say that $\mathrm{cmf} \neq 0$, then the open, driven kinetic system eventually settles into a nonequilibrium steady state $\mathbf{x}^{s s}$ (or some more complex beheviors like oscillations, ) with $\operatorname{cmf}\left(\mathbf{x}^{s s}\right)=\sigma\left(\mathbf{x}^{s s}\right)>0$.

Kinetics with complex balance. The emergence of $\varphi^{s s}(\mathbf{x})$ given in Eq. 6 is highly abstract. One example of this, thanks to the recent work of Anderson et. al [21], is when the kinetic system is complex balanced, a notion introduced by Horn and Jackson in 1972 [22]. This is a class of models which contains detailed balance, all unimolecular reaction networks, as well as many open, driven, nonlinear chemical systems. In this case, it can be shown that the kinetics equation (4) has a unique steady state $\mathrm{x}^{s s}$ and

$$
\varphi^{s s}(\mathbf{x})=\sum_{i=1}^{N} x_{i} \ln \left(\frac{x_{i}}{x_{i}^{s s}}\right)-x_{i}+x_{i}^{s s},
$$

which is a solution to (6), and the limit of $-V^{-1} \ln p_{V}^{s s}(V \mathbf{x})$ when $V$ tends infinity.

We note that for this large class of chemical kinetics, linear and nonlinear, closed and open, equilibrium and nonequilibirum, there is a generic, universal expression for the chemical energy $\varphi^{s s}(\mathbf{x})$, Eq. 10, which has aleady been in the chemical literature. This illustrates the important idea that macroscopic emergent behavior, such as thermodynamics, should be independent of the underlying details of the kinetics.

Discussion. In summary, macroscopic chemical thermodynamics can have a rigous mesoscopic, statistical, reaction kinetic foundation. Chemical free energy, which is a generalization of Gibbs' equilibrium free energy, is an emergent quantity in the macroscopic limit. It actually has a free energy balance equation which is different from Newtonian mechanical energy conservation as well as Helmholtz-Boltzmann's derivation of the First Law of Thermodynamics based on their mechanical theory of heat. This free energy balance equation is applicable to closed and driven chemical reaction kinetic systems under isothermal conditions. This chemical theory of reaction kinetics also provides a concrete example for P. W. Anderson's structure of scientific laws [23]: Macroscopic laws are emergent behaviors from the dynamics of a level below; such laws are insensitive to a large extend the details of the dynamics. It also provides us a tantalizing possibility for high-energy theoretical physics: The universe is made of particles of many kinds and types; the laws that governs their creation, annihilation, transformation, and interactions are the fundamental theory of quantum world. Yet, 
at the macroscopic, cosmological scale, one expects emergent laws that govern its macroscopic dynamics in terms of a "mysterious" force. Such a force should satisfy an equation like our Eq. 6. Currently, there is an active research program in mathematics that studies this type of nonlinear equations [24] that includes (6) as well as Ricci flow, the mathematical structure of Einstein's space-time.

Perhaps, chemical science, in addition to providing the living world with tangible materials and useful energy, can also offer some fundamental insights on how the universe works.

\section{References}

[1] Lewis, G. N. and Randall, M. (1923) Thermodynamics and the Free Energy of Chemical Substances, McGraw-Hill, New York.

[2] Campisi, M. (2005) On the mechanical foundations of thermodynamics: The generalized Helmholtz theorem. Stud. History Philos. Mod. Phys. 36, 275290.

[3] Gallavotti, G. (1999) Statistical mechanics: A short treatise, Springer, Berlin.

[4] Moerner, W. E. (2015) Single-molecule spectroscopy, imaging, and photocontrol: Foundations for super-resolution microscopy (Nobel lecture). Angew. Chem. Int. Ed. 54, 8067-8093.

[5] Beard, D. A. and Qian, H. (2008) Chemical Biophysics: Quantitative Analysis of Cellular Systems, Cambridge Univ. Press, U.K.

[6] Kurtz, T. G. (1972) The relationship between stochastic and deterministic models for chemical reactions. J. Chem. Phys. 57, 2976-2978.

[7] Jarzynski, C. (2011) Annu. Rev. Cond. Matt. Phys. 2, 329-351.

[8] Crooks, G. (1999) Phys. Rev. E 60, 2721-2726.

[9] Ge, H. and Qian, H. (2010) The physical origins of entropy production, free energy dissipation and their mathematical representations. Phys. Rev. E 81, 051133.

[10] Esposito, M. and van den Broeck, C. (2010) Three detailed fluctuation theorems. Phys. Rev. Lett. 104, 090601.

[11] Qian, H. (2013) A decomposition of irreversible diffusion processes without detailed balance. J. Math. Phys. 54, 053302. 
[12] Ge, H. and Qian, H. (2013) Heat dissipation and nonequilibrium thermodynamics of quasi-steady states and open driven steady state. Phys. Rev. E. 87, 062125.

[13] Bergmann, P. G. and Lebowitz, J. L. (1955) New approach to nonequilibrium processes. Phys. Rev. 99, 578-587.

[14] Qian, H. (2001) Relative entropy: Free energy associated with equilibrium fluctuations and nonequilibrium deviations. Phys. Rev. E. 63, 042103.

[15] Ge, H. and Qian, H. (2015) Nonequilibrium thermodynamic formalism of nonlinear chemical reaction systems with Waage-Guldberg's law of mass action. Preprint.

[16] Ge, H. and Qian, H. (2015) Nonequilibrium thermodynamic formalism of nonlinear chemical reaction systems: II: General rate law. Preprint.

[17] Kurtz, T. G. (1978) Strong approximation theorems for density dependent Markov chains. Stoch. Proc. Appl. 6, 223-240.

[18] Hu, G. (1986) Lyapounov function and stationary probability distributions. Zeit. Phys. B Condensed Matter 65, 103-106.

[19] Touchette, H. (2009) The large deviation approach to statistical mechanics. Physics Reports 478, 1-69.

[20] Lewis, G. N. (1925) A new principle of equilibrium. Proc. Natl. Acad. Sci. USA 11 179-183.

[21] Anderson, D. F., Craciun, G., Gopalkrishnan, M. and Wiuf, C. (2015) Lyapunov functions, stationary distributions, and non-equilibrium potential for reaction networks. Bullet. Math. Biol., 77, 1744-1767.

[22] Horn, F. and Jackson, R. (1972) General mass action kinetics. Arch. Rational Mech. Anal. 47, 81-116.

[23] Anderson, P. W. (1972) More is different: Broken symmetry and the nature of the hierarchical structure of science. Science 177, 393-396.

[24] Villani, C. (2009) Optimal Transport, Old and New, Springer-Verlag, New York. 